\title{
Searching for Maximum Cliques with Ant Colony Optimization
}

\author{
Serge Fenet and Christine Solnon \\ LIRIS, Nautibus, University Lyon I \\ 43 Bd du 11 novembre, 69622 Villeurbanne cedex, France \\ \{sfenet,csolnon\}@bat710.univ-lyon1.fr
}

\begin{abstract}
In this paper, we investigate the capabilities of Ant Colony Optimization (ACO) for solving the maximum clique problem. We describe Ant-Clique, an algorithm that successively generates maximal cliques through the repeated addition of vertices into partial cliques. ACO is used to choose, at each step, the vertex to add. We illustrate the behaviour of this algorithm on two representative benchmark instances and we study the impact of pheromone on the solution process. We also experimentally compare Ant-Clique with GLS, a Genetic Local Search approach, and we show that Ant-Clique finds larger cliques, on average, on a majority of DIMACS benchmark instances, even though it does not reach the best known results on some instances.
\end{abstract}

\section{Introduction}

The maximum clique problem is a classical combinatorial optimization problem that has important applications in different domains, such as coding theory, fault diagnosis or computer vision. Given a non-oriented graph $G=(V, E)$, such that $V$ is a set of vertices, and $E \subseteq V \times V$ is a set of edges, a clique is a set of vertices $\mathcal{C} \subseteq V$ such that every couple of distinct vertices of $\mathcal{C}$ is connected with an edge in $G$, i.e., the subgraph induced by $\mathcal{C}$ is complete. A clique is partial if it is strictly included in another clique; otherwise it is maximal. The goal of the maximum clique problem is to find a clique of maximum cardinality.

This problem is one of the first problems shown to be NP-complete, and moreover, it does not admit a polynomial-time approximation algorithm (unless $\mathrm{P}=\mathrm{NP}$ ) [2]. Hence, complete approaches - usually based on a branch-and-bound tree search - become intractable when the number of vertices increases, and much effort has recently been directed on heuristic incomplete approaches. These approaches leave out exhaustivity and use heuristics to guide the search towards promising areas of the search space. In particular, [6] describes three heuristic algorithms obtained as instances of an evolutionary algorithm scheme, called GLS, that combines a genetic approach with local search.

In this paper, we investigate the capabilities of another bio-inspired metaheuristic - Ant Colony Optimization (ACO) - for solving the maximum clique problem. In Section 2, we describe Ant-Clique, an ACO algorithm for the maximum clique problem. Basically, this algorithm uses a sequential greedy heuristic, 
and generates maximal cliques through the repeated addition of vertices into partial cliques. ACO is introduced as a heuristic for choosing, at each step, the vertex to enter the clique: this vertex is chosen with respect to a probability that depends on pheromone trails laying between it and the clique under construction, while pheromone trails are deposited by ants proportionally to the quality of the previously computed cliques. In Section 3, we illustrate the behavior of this algorithm on two representative benchmark instances: we study the impact of pheromone, and we show that the solution process is strongly improved by ACO, without using any other local heuristic nor local search. In Section 4, we experimentally compare Ant-Clique with GLS [6], a genetic local search approach, and we show that Ant-Clique finds larger cliques, on average, on a wide majority of benchmark instances of the DIMACS library, eventhough it does not reach the best known results on some benchmark instances.

\section{Description of Ant-Clique}

The Ant Colony Optimization (ACO) metaheuristic is a bio-inspired approach that has been used to solve different hard combinatorial optimization problems $[3,4]$. The main idea of ACO is to model the problem as the search for a minimum cost path in a graph. Artificial ants walk through this graph, looking for good paths. Each ant has a rather simple behaviour so that it will typically only find rather poor quality paths on its own. Better paths are found as the emergent result of the global cooperation among ants in the colony. This cooperation is performed in an indirect way through pheromone laying.

The proposed ACO algorithm for searching for maximum cliques, called AntClique is sketched below:

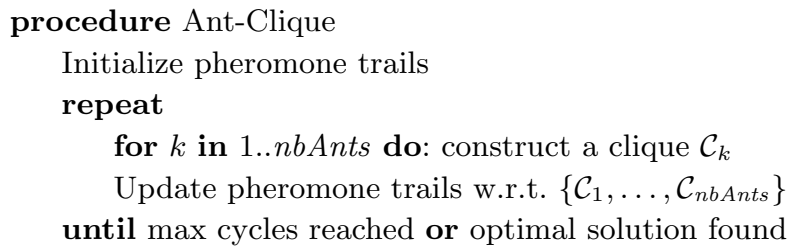

Pheromone trail initialization: Ants communicate by laying pheromone on the graph edges. The amount of pheromone on edge $\left(v_{i}, v_{j}\right)$ is noted $\tau\left(v_{i}, v_{j}\right)$ and represents the learnt desirability for $v_{i}$ and $v_{j}$ to belong to a same clique.

As proposed in [9], we explicitly impose lower and upper bounds $\tau_{\min }$ and $\tau_{\max }$ on pheromone trails (with $0<\tau_{\min }<\tau_{\max }$ ). The goal is to favor a larger exploration of the search space by preventing the relative differences between pheromone trails from becoming too extreme during processing.

We have considered two different ways of initializing pheromone trails:

1. Constant initialization to $\tau_{\max }$ : such an initialization achieves a higher exploration of the search space during the first cycles, as all edges nearly have the same amount of pheromone during the first cycles [9]. 
2. Initialization with respect to a preprocessing step: the idea is to first compute a representative set of maximal cliques without using pheromone - thus constituting a kind of sampling of the search space - and then to select from this sample set the best cliques and use them to initialize pheromone trails. This preprocessing step introduces two new parameters: $\epsilon$, the limit rate on the quality improvement of the sample set of cliques, and $n_{B e s t}$, the number of best cliques that are selected from the sample set in order to initialize pheromone trails. More details can be found in [8].

Construction of cliques by ants: ants first randomly select an initial vertex, and then iteratively choose vertices to be added to the clique - within a set of candidates that contains all vertices that are connected to every clique vertex:

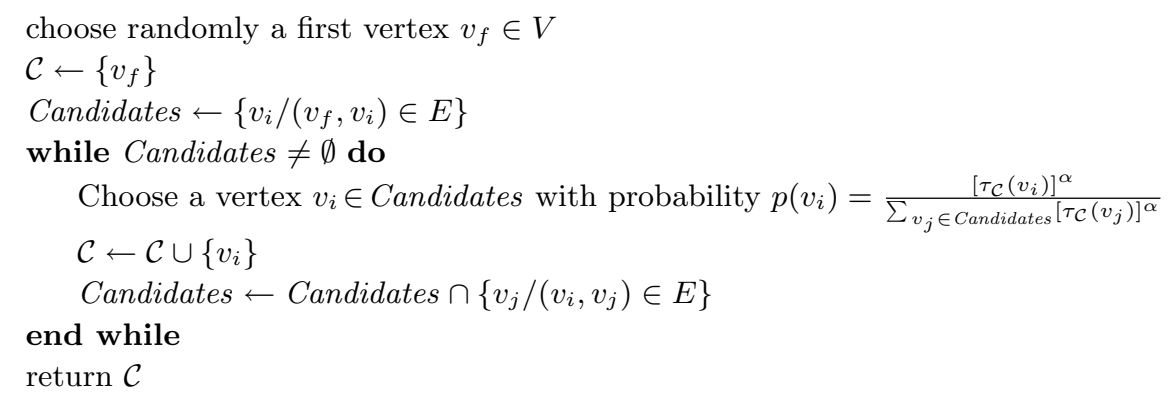

The choice of a vertex $v_{i}$ within the set of candidates is made with respect to a probability that depends on a pheromone factor $\tau_{\mathcal{C}}\left(v_{i}\right)$. A main difference with many ACO algorithms is that this factor does not only depend on the pheromone trail between the last added vertex in $\mathcal{C}$ and the candidate vertex $v_{i}$, but on all pheromone trails between $\mathcal{C}$ and $v_{i}{ }^{1}$, i.e., $\tau_{\mathcal{C}}\left(v_{i}\right)=\sum_{v_{j} \in \mathcal{C}} \tau\left(v_{i}, v_{j}\right)$.

One should remark that the probability of choosing a vertex $v_{i}$ only depends on a pheromone factor, and not on some heuristic factor that locally evaluates the quality of the candidate vertex, as usually in ACO algorithms. Actually, we have experimented a heuristic proposed in [1], the idea of which being to favor vertices with larger degrees in the subgraph induced by the partial clique under construction. The underlying motivation is that a larger degree implies a larger number of candidates after the vertex is added to the current clique. When using no pheromone (or at the beginning of the search, when all pheromone trails have the same value), this heuristic actually allows ants to construct larger cliques than a random choice. However, when combining it with pheromone learning, we have noticed that after a hundred or so cycles, we obtain larger cliques without using the heuristic than when using it.

Updating pheromone trails: After each ant has constructed a clique, pheromone trails are updated according to ACO: first, all pheromone trails are decreased

\footnotetext{
${ }^{1}$ This pheromone factor $\tau_{\mathcal{C}}\left(v_{i}\right)$ is computed in an incremental way: at the beginning, when the clique $\mathcal{C}$ only contains one vertex $v_{f}, \tau_{\mathcal{C}}\left(v_{i}\right)$ is initialized to $\tau\left(v_{f}, v_{i}\right)$; then, each time a new vertex $v_{k}$ is added to the clique, $\tau_{\mathcal{C}}\left(v_{i}\right)$ is incremented with $\tau\left(v_{k}, v_{i}\right)$.
} 
in order to simulate evaporation, i.e., for each edge $\left(v_{i}, v_{j}\right) \in E$, the quantity of pheromone laying on it, $\tau\left(v_{i}, v_{j}\right)$, is multiplied by an evaporation parameter $\rho$ such that $0 \leq \rho \leq 1$; then, the best ant of the cycle deposits pheromone, i.e., let $\mathcal{C}_{k}$ be the largest clique built during the cycle, and $\mathcal{C}_{\text {best }}$ be the largest clique built since the beginning of the run, for each couple of vertices $\left(v_{i}, v_{j}\right) \in \mathcal{C}_{k}$, we increment the quantity of pheromone laying on it, $\tau\left(v_{i}, v_{j}\right)$, by $1 /\left(1+\left|\mathcal{C}_{\text {best }}\right|-\left|\mathcal{C}_{k}\right|\right)$.

\section{Experimental study of Ant-Clique}

As usually in ACO algorithms, the behaviour of Ant-Clique depends on its parameters, and more particularly on $\alpha$, the pheromone factor weight, and $\rho$, the evaporation rate. Indeed, diversification can be emphasized both by decreasing $\alpha$, so that ants become less sensitive to pheromone trails, and by increasing $\rho$, so that pheromone evaporates more slowly. When increasing the exploratory ability of ants in this way, one usually finds better solutions, but as a counterpart it takes longer time to find them. This is illustrated in figure 1 on two DIMACS benchmark instances.

On this figure, one can first note that, for both instances, when $\alpha=0$ and $\rho=1$, cliques are much smaller: in this case, pheromone is totally ignored and the resulting search process performs as a random one so that after 500 or so cycles, the size of the largest clique nearly stops increasing. This shows that pheromone actually improves the solution process with respect to a pure random algorithm.

When considering instance gen_400_P0.9_75, we can observe that, when $\alpha$ increases or $\rho$ decreases, ants converge quicker towards the optimal solution: it is found around cycle 650 when $\alpha=1$ and $\rho=0.995$, around cycle 400 when $\alpha=2$ and $\rho=0.995$, around cycle 260 when $\alpha=2$ and $\rho=0.99$, and around cycle 200 when $\alpha=3$ and $\rho=0.985$. Actually, this instance is relatively easy to solve, regardless of the parameter tuning. In this case, one has better choose parameter values that favor a quick convergence such as $\alpha=3$ and $\rho=0.985$.

However, when considering instance C500.9, which is more difficult, one can see that the setting of $\alpha$ and $\rho$ let us balance between two main tendencies. On one hand, when favoring exploration, the quality of the final solution is better, but the time needed to converge on this value is also higher. On the other hand, when favoring convergence, ants find better solutions during the first cycles, but after 500 or so cycles, too many of them have converged on over-marked pheromone trails and the exploration behavior is inhibited. To summarize, one has to choose parameters depending on the availability of time for solving: for 300 cycles, one has to choose $\alpha=3$ and $\rho=0.985$; for 800 cycles one has to choose $\alpha=2$ and $\rho=0.990$; and for more available time one has to choose $\alpha=2$ and $\rho=0.995$. Note that with $\alpha=1$ and $\rho=0.995$, pheromone's influence is too weak and convergence is too slow: the system won't reach optimal solution in acceptable time. 

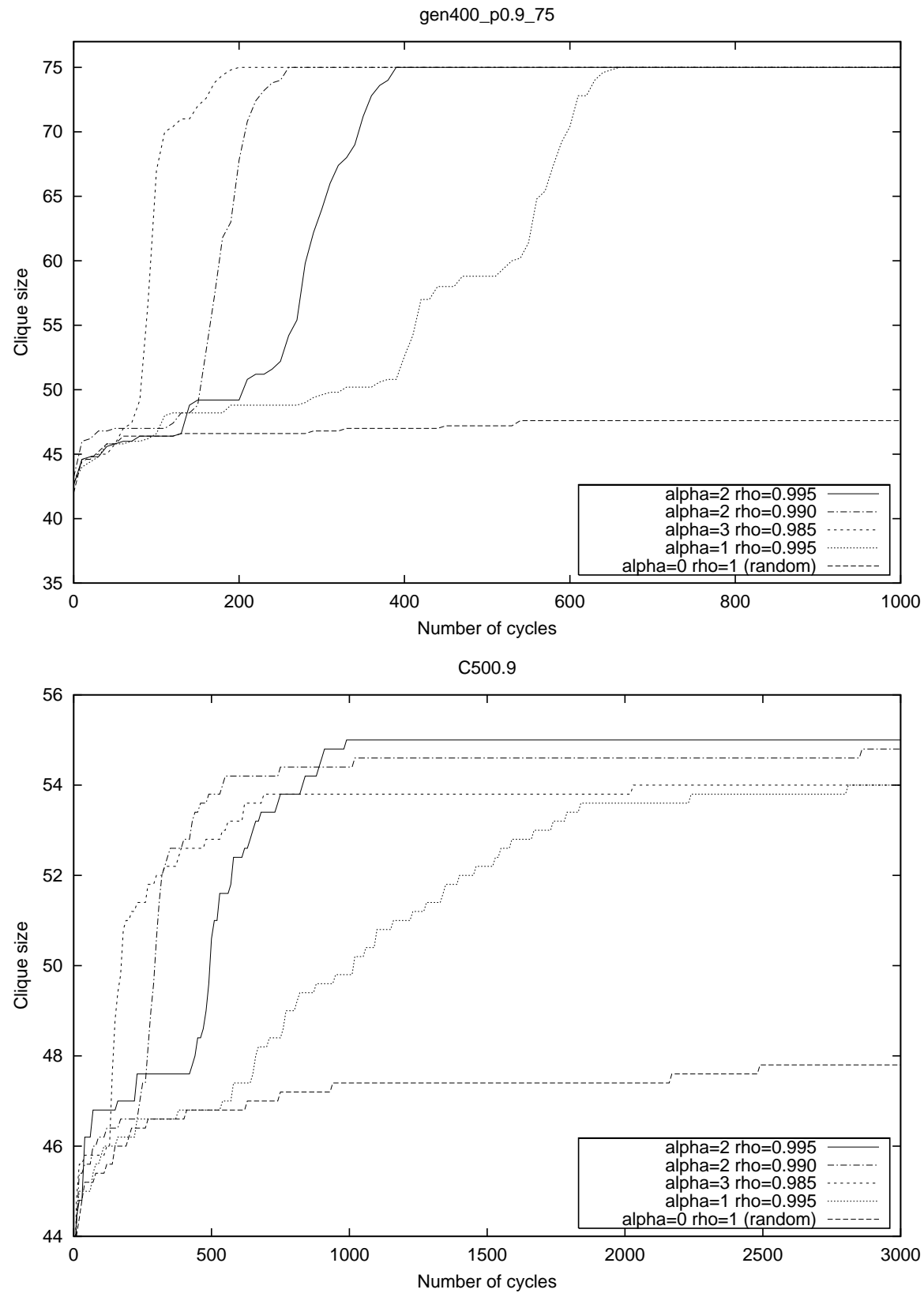

Fig. 1. Influence of pheromone on the solution process: each curve plots the evolution of the size of the largest clique (average on 5 runs), when the number of cycles increases, for a given setting of $\alpha$ and $\rho$. The other parameters have been set to nbAnts $=15$, $\tau_{\min }=0.01, \tau_{\max }=4$, and no preprocessing. The upper curves correspond to the DIMACS instance gen_400_P0.9_75 - that is a rather easy one- and the lower curves to $C 500.9$ - that is more difficult. 


\section{Experimental comparison of Ant-Clique with GLS}

Genetic Local Search: We compare Ant-Clique with genetic local search (GLS) [6], an evolutionary approach that combines a genetic approach with local search. GLS generates successive populations of maximal cliques from an initial one by repeatedly selecting two parent cliques from the current population, recombining them to generate two children cliques, applying local search on children to obtain maximal cliques, and adding to the new population the best two cliques of parents and children. GLS can be instanciated to different algorithms by modifying its parameters. In particular, [6] compares results obtained by the three following instances of GLS: GENE performs genetic local search (the population size is set to 10 , the number of generations to 2000 , and mutation and crossover rates to 0.1 and 0.9 ); ITER performs iterated local search, starting from one random point (the population size is set to 1 , the number of generations to 20000, and mutation and crossover rates are null); and MULT performs multi-start local search, starting from a new random point at each time (the population size is set to 20000, the number of generations, mutation and crossover rates to 0 ).

Experimental Setup: Ant-Clique has been implemented in C and run on a 350 $\mathrm{MHz}$ G4 processor. In all experiments, we have set $\alpha$ to $2, \rho$ to $0.995, \tau_{\min }$ to 0.01 , $\tau_{\max }$ to 4 , and the maximum number of cycles to 3000 . We first report results obtained with 7 ants and no preprocessing. In this case, Ant-Clique constructs 21000 cliques so that this allow a fair comparison with GLS that constructs 20000 cliques. We also report results obtained when increasing the number of ants to 30 , and when using a preprocessing step to initialize pheromone trails (as described in section 2). When a preprocessing step is used, we have set $\epsilon$ to 0.001 and $n_{\text {Best }}$ to 300 .

Test Suite: We consider the 37 benchmark instances proposed by the DIMACS organisers and available at http://dimacs.rutgers.edu/Challenge. Cn.p and DSJC n.p graphs have $n$ vertices; Mann_a 27, 45 and 81 graphs respectively have 378,1035 and 3321 vertices; brockn_ $m$ graphs have $n$ vertices; gen $x x x \_p 0.9 y y$ graphs have $x x x$ vertices and maximum cliques of size yy; hamming $8-4$ and hamming10-4 respectively have 256 and 1024 vertices; keller 4, 5 and 6 graphs respectively have 171, 776 and 3361 vertices; and p_hat $n-m$ graphs have $n$ vertices.

Comparison of Clique Sizes: Table 1 compares the sizes of the cliques found by Ant-Clique and GLS (note that for GLS, we report the results given in [6] for the GLS instance that obtained the best average results). On this table, one can first note that GLS clearly outperforms Ant-Clique on the three MANN instances: on these instances, ACO deteriorates the solution process, and the average size of the constructed cliques at each cycle decreases when the number of cycles increases. Further work will concern the study of the fitness landscape of these instances, in order to understand these bad results.

When comparing average results of Ant-Clique with 7 ants and no preprocessing with GLS, we note that Ant-Clique outperforms GLS on 23 instances, 
Table 1. Results for DIMACS benchmarks. For each instance, the best known result is presented between parenthesis in first column. The table then displays the results obtained by Ant-Clique (with preprocessing and 7 ants, without preprocessing and 7 ants, and without preprocessing and 30 ants) and reports the best, average and standard deviation of the maximum size cliques constructed over 25 runs. Finally, the table reports results given for GLS in [6]: we display the best, average and standard deviation for the GLS algorithm that obtained the best average results (I for Iter, G for Gene and M for Mult). For each instance, best average results are in bold font.

\begin{tabular}{|c|c|c|c|c|c|c|c|c|}
\hline \multirow{4}{*}{ Graph } & \multicolumn{6}{|c|}{ Ant-Clique } & \multirow[b]{4}{*}{ best } & \multirow{3}{*}{ GLS } \\
\hline & \multirow{2}{*}{\multicolumn{2}{|c|}{$\begin{array}{c}\text { with preproc } \\
7 \text { ants }\end{array}$}} & \multicolumn{4}{|c|}{ without preprocessing } & & \\
\hline & & & & 7 ants & & 30 ants & & \\
\hline & best & $\operatorname{avg}(\mathrm{sdv})$ & best & $\operatorname{avg}(\mathrm{sdv})$ & best & $\operatorname{avg}(\mathrm{sdv})$ & & $\operatorname{avg}(\mathrm{sdv})$ \\
\hline C125.9(34) & & 34.0(0.0) & 34 & $\mathbf{3 4 . 0}(0.0)$ & 34 & $\mathbf{3 4 . 0}(0.0)$ & 34 & $\mathbf{3 4 . 0}(0.0)$ \\
\hline $\mathrm{C} 250.9(44)$ & 44 & $44.0(0.0)$ & 44 & $44.0(0.2)$ & 44 & $44.0(0.0)$ & 44 & $43.0(0.6) \quad \mathrm{I}$ \\
\hline C500.9(57) & 56 & $54.3(1.1)$ & 56 & $55.0(0.8)$ & 56 & $\mathbf{5 5 . 5}(0.7)$ & 55 & $52.7(1.4) \quad \mathrm{I}$ \\
\hline C1000.9(68) & 65 & $63.5(1.2)$ & 66 & $63.6(1.4)$ & 67 & $\mathbf{6 5 . 0}(1.0)$ & 66 & $61.6(2.1) \mathrm{G}$ \\
\hline C2000.9(78) & 70 & $68.5(1.2)$ & 72 & $68.5(1.9)$ & 73 & 70.0( & 70 & (1.2) I \\
\hline DSJC500.5(14) & 13 & $\mathbf{1 2 . 9}(0.3)$ & 13 & $12.7(0.5)$ & 13 & $\mathbf{1 2 . 9}(0.3)$ & 13 & .4) $\mathrm{G}$ \\
\hline DSJC1000.5(15) & 15 & $14.0(0.5)$ & 15 & $13.9(0.6)$ & 15 & $\mathbf{1 4 . 0}(0.2)$ & 14 & $13.5(0.5) \mathrm{I}$ \\
\hline C2000.5(16) & 15 & $14.9(0.3)$ & 16 & $14.6(0.6)$ & 16 & $\mathbf{1 5 . 0}(0.3)$ & 15 & $14.2(0.4)$ \\
\hline $\mathrm{C} 4000.5(18)$ & 17 & $15.8(0.6)$ & 16 & $15.5(0.5)$ & 17 & $15.9(0.4)$ & 16 & $15.6(0.5)$ \\
\hline MANN_a27(126) & 126 & $125.6(0.3)$ & 126 & $124.8(0.4)$ & 126 & $125.5(0.5)$ & 126 & $126.0(0.0)$ \\
\hline MANN_a45(345) & 340 & $338.9(0.7)$ & 338 & $336.4(0.6)$ & 341 & $339.2(0.7)$ & 345 & 343.1(0.8) \\
\hline MANN_a81(1098) & 10921 & 1090.6(0.7) & 1087 & $1086.3(0.5)$ & 10921 & $1089.4(0.7)$ & 1098 & $1097.0(0.4)$ I \\
\hline brock200_2(12) & 12 & $11.9(0.3)$ & 12 & $11.9(0.3)$ & 12 & $\mathbf{1 2 . 0}(0.0)$ & 12 & $\mathbf{1 2 . 0}(0.0) \mathrm{M}$ \\
\hline brock200_4(17) & 17 & $16.4(0.5)$ & 16 & $16.0(0.0)$ & 17 & & 7 & \\
\hline brock400_2(29) & 25 & $24.5(0.5)$ & 25 & 24.4 & 29 & & 5 & .7) I \\
\hline brock400_4(33) & 33 & $25.3(3.0)$ & 25 & 24.1 & 33 & & 2 & .8) G \\
\hline brock800_2(21) & 21 & 19.7 & 21 & 19.6 & 21 & & 20 & \\
\hline brock800_4(21) & 20 & $19.3(0.5)$ & 21 & $19.6(0.6)$ & 20 & & 20 & .4) I \\
\hline gen200...44(44) & 44 & $\mathbf{4 4 . 0}(0.0)$ & 44 & $42.6(1.9)$ & 44 & $43.8(0.8)$ & 44 & 1.6) $\mathrm{G}$ \\
\hline gen $200 \ldots 55(55)$ & 55 & $\mathbf{5 5 . 0}(0.0)$ & 55 & $\mathbf{5 5 . 0}(0.0)$ & 55 & 55.0( & & \\
\hline gen $400 \ldots 55(55)$ & 52 & $51.6(0.5)$ & 52 & $51.5(0.5)$ & 52 & & & .2) G \\
\hline gen400...65(65) & 65 & $\mathbf{6 5 . 0}(0.0)$ & 65 & $\mathbf{6 5 . 0}(0.0)$ & 65 & & 5 & 7.4) G \\
\hline gen $400 \ldots 75(75)$ & 75 & $\mathbf{7 5 . 0}(0.0)$ & 75 & $\mathbf{7 5 . 0}(0.0)$ & 75 & $\mathbf{7 5 . 0}(0.0)$ & 75 & $62.7(12.3) \quad \mathrm{I}$ \\
\hline hamming8-4(16) & 16 & $\mathbf{1 6 . 0}(0.0)$ & 16 & $\mathbf{1 6 . 0}(0.0)$ & 16 & $\mathbf{1 6 . 0}(0.0)$ & 16 & 16.0 $(0.0) \mathrm{G}$ \\
\hline hamming10-4(40) & 40 & $38.3(1.0)$ & 40 & $38.4(1.5)$ & 40 & $38.3(1$ & 40 & \\
\hline & 11 & & 11 & 11.0 & 11 & & 11 & $0.0) \mathrm{G}$ \\
\hline & 27 & & 27 & & 27 & & 27 & \\
\hline keller6 & 54 & & 53 & 51.3 & 55 & 53. & 56 & 1.8) I \\
\hline hat $300-$ & 8 & & 8 & & 8 & & 8 & \\
\hline p_hat300-2 & 25 & 25.0 & 25 & 25.0 & 25 & 25.0 & 25 & .0) I \\
\hline & 36 & & 36 & 36.0 & 36 & & & \\
\hline & 11 & & 11 & & 11 & & & \\
\hline & 44 & 44.0 & 44 & 44.0 & 44 & & 4 & 43.6 \\
\hline & 62 & & 62 & & 62 & & 62 & 61.8 \\
\hline p_hat1500-1(12) & 11 & $10.9(0.3)$ & 12 & $11.2(0.4)$ & 12 & & 11 & $10.8(0.4) \mathrm{G}$ \\
\hline & 65 & 64.9 & 65 & & 65 & & 65 & $63.9(2.0) \quad \mathrm{I}$ \\
\hline p_hat1500-3(94) & 94 & $92.9(0.4)$ & 94 & $92.8(0.5)$ & 94 & $\mathbf{9 3 . 1}(0.3)$ & 94 & $93.0(0.8) \quad \mathrm{I}$ \\
\hline
\end{tabular}


whereas GLS outperforms Ant-Clique on 8 instances. Then, when increasing the number of ants from 7 to 30, the average results found by Ant-Clique are improved for 24 instances, whereas they are deteriorated for 2 instances. When further increasing the number of ants, Ant-Clique can still improve its performances on the hardest instances. For example, for keller6 and with 100 ants, Ant-Clique found a clique of size 59, and in average found cliques of size 54.1 instead of 53.1 with 30 ants and 51.3 with 7 ants. Finally, one can also note that, when fixing the number of ants to 7 , the preprocessing step improves average solutions' quality for 18 instances, whereas it deteriorates it for 4 instances.

CPU times: Table 2 reports the number of cycles and the time spent by AntClique on a $350 \mathrm{MHz}$ G4 processor to find best solutions. The table also displays the time spent by the best of the 3 GLS algorithm on a Sun UltraSPARC-II $400 \mathrm{MHz}$ as reported in $[6]^{2}$.

First, note that, within a same set of instances, time spent by Ant-Clique grows quadratically with respect to the number of vertices of the graph, whereas time spent by GLS increases more slowly (even though CPU times are not exactly comparable, as we used different processors). For instance, when considering C125.9, C500.9 and C2000.9, that respectively have 125, 500 and 2000 vertices, Ant-Clique with 7 ants and no preprocessing respectively spent $0.4,34.9$ and 689.3 seconds whereas ITER respectively spent $0.5,2.7$ and 24.8 seconds.

Note also that when increasing the number of ants from 7 to 30 , the number of cycles is nearly always decreased (for all instances but 3 ) as the quality of the best computed clique at each cycle is improved. However, as the time needed to compute one cycle also increases, Ant-Clique with 30 ants is quicker than AntClique with 7 ants for 15 instances only (those for which the number of cycles is strongly decreased), whereas it is slowler for 21 instances.

Finally, note that when initializing pheromone trails with a preprocessing step, instead of a constant initialization to $\tau_{\max }$, the number of cycles is nearly always strongly decreased (for all instances but 2). Indeed, this preprocessing step shorten the exploration phase, so that ants start converging much sooner. One can notice that on very easy instances, the best solution is found during preprocessing, or just after, during the first cycles of the pheromone-oriented solving step. Also, on the $3 M A N N$ instances, the best solution is always found during preprocessing as, on these instances, ACO deteriorates solutions quality. However, if the preprocessing step reduces the number of cycles, it is time consuming. Hence, Ant-Clique with preprocessing is quicker than without preprocessing for 24 instances, whereas it is slowler for 13 . This result must be considered with the fact that preprocessing also allowed to improve solutions' quality for 18 instances, whereas it deteriorates them for 4 instances. Hence, on the maximum clique problem, preprocessing often boosts the resolution.

\footnotetext{
${ }^{2}$ When considering the SPEC benchmarks (http://www . specbench.org/), these two computers exhibit approximately the same computing power: for our $350 \mathrm{MHz}$ G4 processor, SPECint95 $=21.4$ and SPECfp95 $=20.4$; for the UltraSPARC-II $400 \mathrm{MHz}$, SPECint95 $=17.2$ and SPECfp95 $=25.9$. Hence, our computer is more powerful for integer processing, but it is less powerful for floating point processing.
} 
Table 2. Time results for DIMACS benchmarks. For each instance, the table displays the results obtained by Ant-Clique (with preprocessing and 7 ants, without preprocessing and 7 ants, and without preprocessing and 30 ants) and reports the number of cycles (average over 25 runs and standard deviation) and the average CPU time needed to find the best solution (on a $350 \mathrm{MHz}$ G4 processor). For ant-Clique with preprocessing, we successively report the time spent for the preprocessing step, the time spent for ACO solving, and the total time. The last column displays the time spent by the best GLS approach (on a Sun UltraSPARC-II $400 \mathrm{MHz}$ ) .

\begin{tabular}{|c|c|c|c|c|c|c|c|c|}
\hline \multirow{4}{*}{ Graph } & \multicolumn{7}{|c|}{ Ant-Clique } & \multirow{4}{*}{$\begin{array}{l}\text { GLS } \\
\text { time }\end{array}$} \\
\hline & \multirow{2}{*}{\multicolumn{3}{|c|}{$\begin{array}{l}\text { with preprocessing } \\
7 \text { ants }\end{array}$}} & \multicolumn{4}{|c|}{ without preprocessing } & \\
\hline & & & & 7 ants & & 30 ants & & \\
\hline & $\mathrm{nbCy}(\mathrm{sdv})$ & $\mathrm{t} \_\mathrm{P}+\mathrm{t} \_\mathrm{S}=$ & time & $\mathrm{nbCy}(\mathrm{sdv})$ & time & $\mathrm{nbCy}(\mathrm{sdv})$ & time & \\
\hline C125.9 & $93(61)$ & $1.0+0.2=$ & 1.2 & $219(63)$ & 0.4 & $140(49)$ & 0.7 & 0.5 \\
\hline C250.9 & $356(144)$ & $2.2+2.5=$ & 4.7 & $888(523)$ & 6.1 & $467(98)$ & 6.7 & 2.4 \\
\hline C500.9 & $974(614)$ & $4.7+24.5=$ & 29.3 & $1488(654)$ & 34.9 & $1211(603)$ & 49.9 & 2.7 \\
\hline C1000.9 & $1730(672)$ & $12.9+154.6=$ & 167.6 & $1972(571)$ & 161.2 & $1712(602)$ & 202.2 & 12.4 \\
\hline C2000.9 & $2065(648)$ & $3.8+672.9=$ & 696.7 & $2281(498)$ & 689.3 & $2041(487)$ & 749.5 & 24.8 \\
\hline DSJC500.5 & $25(614)$ & $0.9+4.5=$ & 5.4 & $1049(952)$ & 12.9 & $(375)$ & 8.8 & 2.1 \\
\hline DSJC1000.5 & $495(612)$ & $1.8+24.4=$ & 26.2 & 1084(910) & 50.6 & $990(645)$ & 51.3 & 2.3 \\
\hline $\mathrm{C} 2000.5$ & $576(741)$ & $4.2+108.3=$ & 112.5 & $1130(919)$ & 203.1 & $869(615)$ & 159.5 & 2.3 \\
\hline C4000.5 & $5(745)$ & $7.6+377.2=$ & 384.8 & $783(826)$ & 551.0 & 1097 & 772.3 & 15.7 \\
\hline MANN_a27 & $0(0)$ & $10.1+0.0=$ & 10.1 & $187(630)$ & 7.8 & $171(455)$ & 21.3 & 15.6 \\
\hline MA & $0(0)$ & $+0.0=$ & 67.7 & $14(14)$ & 4.4 & $233(508)$ & 231.3 & 54.4 \\
\hline MANN_a81 & $0(0)$ & $84.6+0.0=$ & 84.6 & $25(23)$ & 97.0 & $7(0)$ & 93.6 & 693.9 \\
\hline brock200_2 & $322(718)$ & $0.1+0.6=$ & 0.7 & $265(492)$ & 0.4 & $80(113)$ & 0.2 & 2.7 \\
\hline 200_4 & 7(197) & & 0.5 & $424(124)$ & 0.9 & 622 & 2.3 & 2.8 \\
\hline $\mathrm{ck} 400 \_2$ & $813(768)$ & $-10.2=$ & 12.3 & $955(427)$ & 10.6 & & 15.1 & 2.0 \\
\hline ck400_4 & 208(151) & & 4.8 & $945(468)$ & 10.4 & & 12.7 & 1.3 \\
\hline k800_2 & $820(986)$ & $2.3=$ & 33.7 & $1437(626)$ & 52.1 & 1227 & 51.3 & 5.2 \\
\hline brock800_4 & $620(512)$ & $1.4+25.4=$ & 26.8 & $1437(576)$ & 51.9 & $1270(776)$ & 53.4 & 4.1 \\
\hline gen200...44 & $364(165)$ & $1.3+1.7=$ & 3.0 & 1065(531) & 4.7 & $376(146)$ & 4.3 & 1.3 \\
\hline gen $200 \ldots 55$ & $4(5)$ & $2.0+0.0=$ & 2.0 & 203(31) & 0.9 & & 1.5 & 1.4 \\
\hline gen $400 \ldots 55$ & $1423(898)$ & $3.3+24.3=$ & 27.7 & $1382(742)$ & 22.3 & 1086(503) & 35.8 & 3.8 \\
\hline gen $400 \ldots 65$ & 281(137) & $3.8+4.7=$ & 8.5 & $718(158)$ & 11.1 & $412(40)$ & 13.2 & 4.3 \\
\hline gen $400 \ldots 75$ & $49(42)$ & $4.3+0.8=$ & 5.1 & $555(443)$ & 8.8 & $289(22)$ & 9.2 & 4.6 \\
\hline hamming8-4 & $0(1)$ & $0.7+0.0=$ & 0.7 & $134(93)$ & 0.5 & $59(41)$ & 0.3 & 0.0 \\
\hline hamming10-4 & $806(493)$ & $7.9+66.8=$ & 74.6 & $1750(632)$ & 129.9 & $1167(323)$ & 103.2 & 5.3 \\
\hline keller 4 & $1(1)$ & $0.5+0.0=$ & 0.5 & $9(8)$ & 0.0 & $3(2)$ & 0.0 & 0.0 \\
\hline keller5 & $936(775)$ & $4.0+40.1=$ & 44.1 & $1417(500)$ & 55.7 & 1063(598) & 50.2 & 4.0 \\
\hline keller6 & $1489(768)$ & $23.6+1180.0=$ & 1203.6 & $2242(375)$ & 1682.4 & $2112(498) 1$ & 1731.3 & 36.2 \\
\hline p_hat300-1 & $4(4)$ & $0.2+0.0=$ & 0.2 & $173(164)$ & 0.4 & $34(31)$ & 0.1 & 0.4 \\
\hline p_hat300-2 & $25(25)$ & $0.8+0.1=$ & 0.9 & $300(48)$ & 1.2 & $190(47)$ & 1.1 & 0.5 \\
\hline p_hat $300-3$ & $402(248)$ & $1.9+3.1=$ & 5.0 & $718(228)$ & 4.9 & $486(96)$ & 5.5 & 1.5 \\
\hline p_hat700-1 & $206(274)$ & $0.4+3.0=$ & 3.4 & $995(375)$ & 14.1 & $619(310)$ & 9.4 & 2.6 \\
\hline p_hat700-2 & $206(127)$ & $3.1+5.5=$ & 8.6 & $551(86)$ & 12.7 & $452(99)$ & 13.0 & 1.2 \\
\hline p_hat700-3 & $919(664)$ & $5.9+35.8=$ & 41.7 & $1106(438)$ & 38.0 & $1030(347)$ & 50.3 & 4.5 \\
\hline p_hat1500-1 & $461(745)$ & $1.1+31.7=$ & 32.8 & $1488(640)$ & 97.0 & $700(374)$ & 46.3 & 14.2 \\
\hline p_hat1500-2 & 791(419) & $7.0+92.2=$ & 99.2 & $1181(580)$ & 125.8 & $790(220)$ & 94.9 & 12.2 \\
\hline p_hat1500-3 & $945(776)$ & $14.9+152.8=$ & 167.8 & $1360(596)$ & 214.7 & 1071(422) & 195.1 & 7.1 \\
\hline
\end{tabular}




\section{Conclusion}

We have described Ant-Clique, an ACO algorithm for searching for maximum cliques. We have shown through experiments that this metaheuristic is actually well suited to this problem: without using any local heuristic to guide them neither local search technics to improve solutions quality, artificial ants are able to find optimal solutions on many difficult benchmark instances.

However, Ant-Clique does not reach the best known results on all instances. The best current approach is Reactive Local Search (RLS) [1], an approach that combines local search with prohibition-based diversification techniques where the amount of diversification is determined in an automated way through a feedback scheme. In the experiments reported here, Ant-Clique has been able to find the best solution found by RLS on 29 instances over 37 .

Hence, further work will first explore the integration within Ant-Clique of some local search technics such as the one used by RLS. Actually, the best performing ACO algorithms for many combinatorial problems are hybrid algorithms that combine probabilistic solution construction by a colony of ants with local search $[5,9,7]$. Also, Ant-Clique performances may be enhanced by introducing a local heuristic to guide ants. Indeed, as pointed out in Section 2, we already have experimented a first heuristic, borrowed from [1], that has not given any improvement. However, we shall further investigate other heuristics.

\section{References}

1. R. Battiti and M. Protasi. Reactive local search for the maximum clique problem. Algorithmica, 29(4):610-637, 2001.

2. I. Bomze, M. Budinich, P. Pardalos, and M. Pelillo. The maximum clique problem. In D.-Z. Du and P. M. Pardalos, editors, Handbook of Combinatorial Optimization, volume 4. Kluwer Academic Publishers, Boston, MA, 1999.

3. M. Dorigo. Optimization, Learning and Natural Algorithms (in Italian). PhD thesis, Dipartimento di Elettronica, Politecnico di Milano, Italy, 1992.

4. M. Dorigo, G. Di Caro, and L. M. Gambardella. Ant algorithms for discrete optimization. Artificial Life, 5(2):137-172, 1999.

5. M. Dorigo and L.M. Gambardella. Ant colony system: A cooperative learning approach to the traveling salesman problem. IEEE Transactions on Evolutionary Computation, 1(1):53-66, 1997.

6. E. Marchiori. Genetic, iterated and multistart local search for the maximum clique problem. In Applications of Evolutionary Computing, Proceedings of EvoWorkshops2002: EvoCOP, EvoIASP, EvoSTim, volume 2279, pages 112-121. SpringerVerlag, 2002.

7. C. Solnon. Ants can solve constraint satisfaction problems. IEEE Transactions on Evolutionary Computation, 6(4):347-357, 2002.

8. C. Solnon. Boosting ACO with a preprocessing step. In Applications of Evolutionary Computing, Proceedings of EvoWorkshops2002: EvoCOP, EvoIASP, EvoSTim, volume 2279, pages 161-170. Springer-Verlag, 2002.

9. T. Stützle and H.H. Hoos. $\mathcal{M} \mathcal{A} \mathcal{X}-\mathcal{M I N}$ Ant System. Journal of Future Generation Computer Systems, 16:889-914, 2000. 